\title{
Airway accidents in an intensive care unit
}

\author{
Aparna Chatterjee, Saeeda Islam, J. V. Divatia
}

Aim: To document the incidence of airway accidents in the Intensive Care Unit, and to identify the differ-
ence in accident rates between short term and long term intubated patients, and between endotracheal
intubation and tracheostomy. Design: Prospective, observational non-interventional study. Patients and
Methods: All adult patients with either an endotracheal tube or tracheostomy were divided into two groups:
intubation for </= 24 hours \{short-term intubation/ STI\}, and intubation for > 24 hours, Long-term intuba-
tion/ LTI\}. The number of tube days (TDs) were calculated from the day of intubation till the day of extuba-
tion, discharge or death. The following airway accidents were noted: blocked tube (BT), unplanned extubations
(UE), endobronchial intubation (EBI), kinked tube and leaking cuff. Results: 781 patients (1440 tube days)
were studied. 665 patients ( 951 tube days) required an endotracheal tube. 116 patients (489 tube days) had
a tracheostomy. 697 patients ( 697 tube days) required STI while 84 patients ( 743 tube days) required LTI.
Overall there were 55 airway accidents with an incidence of $7.04 \%$ of patients and 3.82 / 100 tube days. The
airway accident rate was 4.02 / 100 TD's and $3.63 / 100$ TD's for STI and LTI, respectively, and $4.21 / 100$
TD's and 3.07 / 100 TD's for endotracheal intuibation and tracheostomy, respectively. Blocked tubes ( 2.15
per 100 TD's) and Unplanned extubations (1.32 per 100 TD's) were the most common airway accidents.
Conclusion: The overall incidence of airway accidents was $3.82 / 100$ TD's and $7.04 \%$ of patients with no
significant difference between STI vs. LTI and endotracheal tube vs. tracheostomy.
Key Words: Airway accidents, Critical Care, blocked tube, unplanned extubations, endobronchial intubation

Control of the airway with the help of a tracheal tube is an important aspect of intensive care. The use of tracheal tubes however is not without complications. The longer a tube stays in-situ, the greater the chances of kinking, blockade and unplanned extubations. All of the above can lead to hypoventilation and hypoxia which are potentially life threatening. The few studies on unplanned extubations have reported an incidence ranging from 0.3 - 30\% ${ }^{1-2}$ Significant proportions of those who have an unplanned extubation tolerate it well and do not require reintubation. ${ }^{6,8}$ Most of these are associated with minimal or no morbidity. ${ }^{4}$ One study of 53 patients however recorded three deaths at the time of unplanned

\section{From:}

Department of Anaesthesia, Critical Care and Pain Management, Tata Memorial Hospital, Mumbai.

Correspondence:

Dr. J. V. Divatia

Department of Anaesthesia, Tata Memorial Hospital, Dr. E. Borges Road, Parel, Mumbai - 400012, India. Email: jdivatia@vsnl.com extubations. $^{2}$ Tata Memorial Hospital is a tertiary level referral center for the comprehensive management of cancer in Mumbai and has a 22-bed ICU. A majority of the ICU admissions are from Surgical oncology, with selective referral from the medical and radiation oncology services. A large number of patients undergoing major head and neck surgery remain intubated in the ICU for airway protection for periods less than 24 hours.

We undertook a study to document the incidence of airway accidents in our ICU, and to identify differences, if any, in airway accident rates between short term and long term-intubated patients and also between those who have an endotracheal tube compared to those with a tracheostomy.

\section{Design}

Prospective observational, non-interventional study carried out in the 22-bed intensive care unit (ICU) over a 
7.5-months period.

\section{Materials and Methods}

Between June 2001 and January 2002, all adult patients admitted to the ICU with either an endotracheal tube or tracheostomy were included in the study. They were divided into those that required intubation for $>4$ hours but $</=24$ hours \{short-term intubation (STI) and those who required a tube for $>24$ hours \{Long-term intubation, (LTI)\}. Tube days (TD) were calculated from the day of intubation till the day of extubation or discharge from, or death in the ICU. The airway accidents occurring during this period were noted. The airway accidents taken into consideration were; blocked tube (BT), unplanned extubation (UE) including both self and accidental extubations, endobronchial intubation (EBI), kinked tube and leaking cuff. Tubes were considered blocked if resistance was felt on passing a suction catheter down the tube or if the lumen was found narrowed at extubation.

All ventilated patients (LTI) were sedated with infusions of midazolam or propofol and morphine or fentanyl. Sedation was intermittently stopped every morning to assess neurological status. Patients undergoing STI (but not ventilated) for airway protection after major head and neck surgery received bolus doses of narcotics and anxiolytics (buprenorphine and midazolam) with or without a background infusion. Restraints are seldom used. Endotracheal tubes were fixed with adhesive tape applied to the lower chin and cheek for orotracheal tubes and to the nose and forehead for nasotracheal tubes. Polyvinyl chloride tubes with high volume-low pressure cuffs were used. PVC, reinforced latex or metal tubes were used for tracheostomies. They were secured either with a tape tied around the neck or sutured in place. Heat and moisture exchange filters (HMEF's) or heated humidifiers were used for humidification ${ }^{12}$ in LTI and intermittent saline nebulisation was used in STI.

The duration for which the tube was in situ, date and time of the airway accident, description of the type of accident, severity of the accident and it's impact on the course of the patient's illness, whether preventable and if needed to be reintubated were noted.

The severity of the airway accident was graded as follows:

Mild: Little or no physiological consequence/ managed by resident on call.

Moderate: Cardio respiratory decompensation/ senior or experienced person required to manage the problem.

Major: Near or actual cardio respiratory arrest/death. Airway accident rate was calculated both in terms of accidents per 100 patients (\% of patients) and accidents per 100 tube days (TD's).

Data was analysed statistically using the chi square test.

\section{Results}

781 patients (1440 tube days) required either an endotracheal tube or a tracheostomy over the 7.5-months period. Details of patients are shown in Figure 1. During this period there were a total of 55 airway accidents with an incidence of $3.8 / 100$ tube days and $7.04 \%$ of patients. The incidence of airway accidents by duration of intubation and the type of airway is as detailed in Table 1. The incidence of specific complications is detailed in Table 2, and Table 3 compares the incidence of complications in patients with STI vs. LTI, and endotracheal intubation (ETI) vs,. Tracheostomy (TR).

On comparing the incidence of overall airway accidents per 100 tube days between STI and LTI, we found no significant difference except in the incidence of blocked tubes, which were found to be higher in the case of STI $(P$ Value $=0.029)$. In STI all blocked tubes were removed while, in LTI the tubes were changed.

There was no difference in the incidence of airway accidents (per 100 TD's) between those with endotracheal

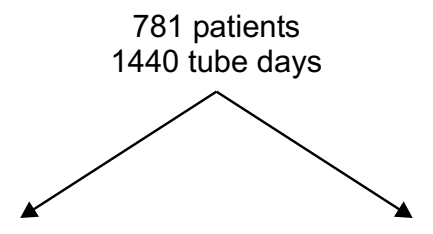

Endotracheal intubation 665 patients / 951 tube days

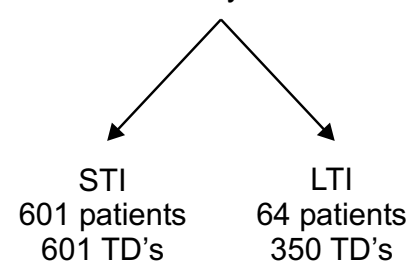

Tracheostomy 116 patients / 489 tube days

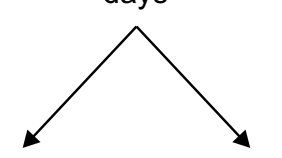

STI 96 patients 96 TD's
LTI 20 patients 393 TD's
Figure 1: The study population and airway accidents 
Table 1: Overall incidence of airway accidents by duration of intubation and type of airway

\begin{tabular}{lccc}
\hline & $\begin{array}{c}\text { Airway Accidents } \\
\text { No. of patients } \\
\text { (tube days) }\end{array}$ & $\begin{array}{c}\text { Incidence } \\
\text { (\% of patients) }\end{array}$ & $\begin{array}{c}\text { Incidence } \\
\text { (Per 100 } \\
\text { tube days) }\end{array}$ \\
Total & 55/781 (1440 TD's) & 7.04 & 3.82 \\
STI & 28/697 (697 TD's) & 4.02 & 4.02 \\
LTI & $27 / 84$ (743 TD's) & 32.14 & 3.63 \\
Type of airway & & & \\
ETI & 40/665 (951 TD's) & 6.02 & 4.21 \\
TR & 15/116 (489 TD's) & 12.93 & 3.07 \\
\hline
\end{tabular}

STI, short-term intubation; LTI, long-term intubation; ETI, endotracheal intubation; TR, tracheostomy.

\begin{tabular}{lccc}
\hline \multicolumn{4}{l}{ Table 2: Incidence of specific complications } \\
\hline Specific & Total \% & I (\% of pts) & I (/100 TDs) \\
Complications & $(\mathbf{n}=\mathbf{5 5})$ & $(\mathbf{n}=\mathbf{7 8 1})$ & $(\mathbf{n = 1 4 4 0 )}$ \\
BT $(n=31)$ & 56.36 & 3.97 & 2.15 \\
UE $(n=19)$ & 34.55 & 2.43 & 1.32 \\
EBI $(n=2)$ & 3.64 & 0.26 & 0.14 \\
Others $(n=3)$ & 5.45 & 0.38 & 0.21 \\
\hline
\end{tabular}

$\mathrm{BT}$, blocked tube; UE, unplanned extubation; EBI, endobronchial extubation.

Table 3: Individual airway accidents per 100 tube days STI vs. LTI \& ETI vs. TR

\begin{tabular}{|c|c|c|c|c|c|c|}
\hline $\begin{array}{l}\text { Airw } \\
\text { Acci }\end{array}$ & $\begin{array}{c}\text { STI } \\
(n=697\end{array}$ & $\begin{array}{c}\text { LTI } \\
(n=743)\end{array}$ & $P$ Value & $\begin{array}{c}\text { ETI } \\
(n=951)\end{array}$ & $\underset{(n=489)}{\text { TR }}$ & $P \mathrm{Va}$ \\
\hline BT & 3.01 & & 0 & & & 0.5 \\
\hline U & & & & & & \\
\hline & 0 & & & & & \\
\hline & 0 & & & & & \\
\hline Total & $4.01(28)$ & $3.63(27)$ & 0.70 & $4.20(40)$ & 3.06 (15) & 5) 0.29 \\
\hline
\end{tabular}

STI, short-term intubation; LTI, long-term intubation; ETI, endotracheal intubation; TR, tracheostomy; BT, blocked tube; UE, unplanned extubation; $\mathrm{EBI}$, endobronchial extubation.

intubation (ETI) as opposed to those with a tracheostomy (TR), except in the case of endobronchial intubations $(\mathrm{EBI})$, the incidence of which was surprisingly found to be higher in the tracheostomy group ( $P$ Value $=0.048$ ).

Endotracheal tubes or tracheostomies that slipped out or were displaced during either change of patients position, sponging or due to patient's agitation were included under the title of unplanned extubations. The incidence of unplanned extubations was $2.43 \%$ overall, and $1.32 /$ 100 tube days.

Of the 55 cases of airway accidents, 48 were minor, 6 were moderate and 1 was a major accident, wherein the patient self-extubated and suffered a cardiac arrest due to hypoxia.

\section{Discussion}

The overall incidence of airway accidents was found to be 3.82/100 tube days. No difference was noted between short-term group (STI) vs long-term intubation group (LTI) or the endotracheal tube group (ETI) vs tracheostomy group (TR).

The incidence of blocked tubes was found to be significantly higher in the group that required short-term intubation (STI). The STI group comprised of patients who had undergone head and neck surgery and were kept under observation in the ICU. These patients were allowed to breathe spontaneously with a nasotracheal tube in situ, received oxygen via a T-piece arrangement and intermittent saline nebulisations to humidify the inspired gases. Most of the tubes were blocked either by blood or thick secretions that made it difficult to pass a suction catheter down these tubes. At extubation some endotracheal tubes were noted to have a significant narrowing of the lumen due to encrustations. We attribute this to inadequate humidification ${ }^{12}$ and have started using continuous saline nebulisations in STI. In LTI, heated humidifiers or heat and moisture exchange filters (HMEF) were used. HMEF's were changed every 24-48 hours. HMEF's are known to provide efficient humidification with no tracheal tube occlusion for upto 48 hours and in some studies for upto 7 days without changing the filter. ${ }^{13,14}$

The rate of unplanned extubations in our study was found to be $1.32 / 100$ tube days (1.32\%). This value is significantly higher in comparison with Kapadia et $\mathrm{al}^{3}$ $(0.14 \%)$ and lower as compared to Epstein et $\mathrm{al}^{4}(1.6 \%)$ and Carrion et $\mathrm{al}^{5}(1.5-2.4 \%)$.

This study was performed to document the incidence of various airway accidents. We did not systematically record data on causes and factors that may have lead to an increased incidence of airway accidents, such as the actual nurse: patient ratio at the time of the accident, time of accident, effect of weaning, and other such factors. The nurse-patient ratio in our ICU ranges from 1:2 to $1: 4$ and depends on the time of the day. We attribute this higher incidence of unplanned extubations to a combination of lack of 1:1 nursing staff in our ICU, inadequate sedation of our intubated patients, and lack of adequate communication and reassurance of our intubated patients as they wake up from sedation.

Unplanned extubation can be a serious complication associated with mortality and is a quality of care con- 
cern. ${ }^{9}$ All the patients who had an unplanned extubation were reintubated. Although few studies have advocated close observation following unplanned extubations as many may not require reintubation. ${ }^{6,7}$

With the use of better tube fixations, changing sedation protocols, ${ }^{10}$ better communication with patients as they wake up from sedation, regular humidification of inspired gases and more vigilance by the medical and nursing staff, ${ }^{11}$ we hope to reduce this incidence of airway accidents. We have implemented the above-mentioned strategies in our ICU in the form of a new airway protocol. An audit to assess this new airway protocol is underway at the moment.

\section{References}

1. Kapadia FN, Bajan KB, Raje KV. Airway accidents in intubated ICU patients: An epidemiological study. Critical Care Medicine 2000;28:659-64.

2. Amato MRP, Barbas CSV, Medeiros DM, et al. Effect of protective ventilator strategy on mortality in acute respiratory distress syndrome. N Eng J Med 1998;338:347-54.

3. Kapadia FN, Bajan KB, Singh S, et al. Changing patterns of airway accidents in intubated patients. Intensive Care Med 2001;27:296-300.

4. Epstein S, Nevins ML, Chung J. Effect of unplanned extubation on outcome of mechanical ventilation. Am J Resp Crit Care Med
2000;161:1912-6.

5. Carrion MI, Ayuso D, Marcos M, et al. Accidental removal of endotracheal and nasogastric tubes and intravascular catheters. Critical Care Med 2000;28:63-6.

6. Coppolo DP, May JJ. Self-extubation: A 12-month experience. Chest 1990;98:165-9.

7. Christie JM, Dethlefsen M. Unplanned endotracheal extubation in the Intensive care unit. J Clin Anaesth 1996;8:289-93.

8. Whelan J, Simpson SQ, Levy H.xtubations. Predictors of successful termination of mechanical ventilatory support. Chest 1994;105:1808-12.

9. Maguire GP, DeLorenzo LJ, Moggio RA. Unplanned extubation in the intensive care unit: A quality-of-care concern. Crit Care Nurs Q 1994;17:40-7.

10. Powers J. A sedation protocol for preventing patient self-extubation. Dimens Crit Care Nurs 1999;18:30-4.

11. Boulain T. Unplanned extubations in the adult intensive care unit. Am J Resp. Crit Care Med 1998;157:1131-7.

12. Joynt GM, Lipman J. Humidification in intensive care. S Afr J Surg 1994;32:23-30.

13. Markowicz P, Richard JD, et al. Safety, efficacy and cost-effectiveness of mechanical ventilation with humidifying filters changed every 48 hours: A prospective randomized study. Crit Care Med 2000;28:665-71.

14. Thomachot L, Leone M, Razzouk K. Randomized clinical trials of extended use of a hydrophobic condenser humidifier: 1 vs 7 days. Crit Care Med 2002;30:232-7. 